\title{
19
}

\section{An Implementation of CMIP/CMISE Conformance Testing System}

Ching-Sung LU, Hudson Chien, Whey-Hsing Tsai, Wan-Ting Chang, Chang-Shing Perng, Ching-Te Lin, Kuang-Jui Cheng, Kuo-Wei Peng, Gary Tzeng

Information Technology Laboratory

Telecommunication Laboratories, Ministry of Transportation and Communications

12, Lane 551, Section 3, Min-tsu Road

Chung-li, Taoyuan 32099, Taiwan

Phone:886-3-4244758. Email:Redfish@twnmoctl.bitnet

\section{Abstract}

CMIP (Common Management Information Protocol)/CMIS (Common Management Information Services) is the international standard for communication between management agents and management managers. It plays the most important role in the communication platform of OSI Network Management. The development of the CMIP/CMISE CTS (Conformance Test System) is based on the CPS Forum Technical Framework Specification [1] and the CMIP/CMISE abstract test suite drafted by the CTS3 NM (Network Management) project [2]. This CTS has been used to perform conformance testing against OSIMIS which was developed by University College London. This paper describes the system development considerations, the system architecture, the test method, and functional modules of the CMIP/CMISE CTS. The supporting tools, TIME (TTCN-based Integrated Management Environment) [10] and TTCN (Tree and Tabular Combined Notation) translator, which can be used to support developing general conformance testing systems, are also described. Finally, the evaluation of the system in testing is given .

\section{Introduction}

\subsection{Importance of OSI Network Management}

We live in an era when computers, telecommunication networks and distributed systems are not only exploding in terms of volume and complexity, but are rapidly becoming as common-place in business as the personal computer. Such rapid changes induce exigent requirements to manage an ever increasing variety of underlying network technologies and applications. Among those various management approaches, OSI Network Management services and protocols are undoubtedly the most promising and important solutions. Within its framework, CMIS (Common Management Information Services) [3] are used by application processes in peer open systems, to exchange information and commands for the purpose of system management. The CMIP (Common Management Information Protocol) [4] specifies protocol elements that may be used to provide the operation and notification services described in CMIS. 


\subsection{Why Conformance Testing}

The objectives of OSI will not be completely achieved until systems can interoperate seamlessly. Conformance test does not guarantee interoperation; however, it gives confidence that an implementation has the required capabilities and that its behavior conforms consistently in representative instances of communication. Besides, it provides a very important evaluation about the protocol product.

\subsection{Test Methodology}

OSI community has already studied various testing methods and their applicability, and defines a guidance to write system-independent conformance test suites. TTCN (Tree and Tabular Combined Notation) language is created for use in the development of standardized ATSs and defined in the Part 3 of the OSI Conformance Testing Methodology and Framework, a multi-part standard, ISO 9646 [9]. Part 1 of 9646: General concepts [7] discusses the various test methods and their applicability to real systems. Part 2 of 9646: ATS ( Abstract Test Suite) Specification [8] provides a guidance for the production of system-independent conformance test suites for one or more OSI standards.

\subsection{Outline of This Paper}

This paper discusses the implementation of CMIP/CMISE CTS and supporting tools. Section 2 discusses the system development considerations. The system architecture is depicted in Section 3. This section introduces the Coordinated Test Method and the system architecture. Section 4 describes the supporting tools, TIME and TTCN translator. Section 5 is the evaluation of the system. Section 6 makes the conclusion. Section 7 lists the terms and abbreviations.

\section{System Development Considerations}

Even though we just implement the CMIP/CMISE CTS up to now, we aim to provide an environment where users can easily develop their ATSs and CTSs. In order to build up such an environment, the following features are provided.

\subsection{Facilitate the Development of ATS and the Derivation of ETS}

In order to provide convenience for further ATS production and CTS development. Two supporting tools, TIME and TTCN translator, have been developed. TIME provides users a convenient environment to write ATSs written in TTCN. Users can also use it to export the TTCN.MP (TTCN Machine Processable) forms which are the input data for TTCN translator, a tool to translate ATS into ETS (Executable Test Suites). 


\subsection{Adopt the Standard ATS}

To provide one consistent report for conformance tests held in different TCs (Test Centers) and such the cross recognition of test results held in different test centers, it is very important that the ATSs are standardized; therefore, our policy is to adopt the standardized ATSs. Fortunately, there is one such ATS for CMIP/CMISE. The CMIP/CMISE Abstract Test Suite developed by the CTS3 Network Management project of European Community has been delivered to AOW, OIW and EWOS, and hopefully will be selected as the standard CMIP/CMISE ATS in the near future.

\subsection{Provide A Consistent User Look-and-Feel and Operation Convention}

Aside from providing a consistent report, there are still many considerations when users implement a CTS. A consistent user look-and-feel reduces not only the investment in training but also the probability of errors which may happen when the operator faces dissimilar systems.

\subsection{Provide A Common Flexible Architecture}

A common flexible and extensible architecture design allows the enhancement of new functionalities and incorporation of new systems. It also increases the probability of components reusability. After studying all these issues, the CPS Forum proposes one specification which defines the user interface, the system functionalities, the architecture and the deposited data for a CPS-registered CTS. We follow the recommendation of CPS Forum and plan to register our system.

\section{System Architecture}

This section discusses the system architecture. Figure 1 shows this architecture and relationship between different components. It depicts only the main functional modules. The left shadow area represents the lower tester, and the right one represents the upper tester.

\subsection{Coordinated Test Method}

There are many discussions about the test methods and their applicability in Part 1 of 9646, and the CMIP/CMISE ATS of CTS3 NM adopts the Coordinated Test Method. Within the Coordinated Test Method, the TMP (Test Management Protocol) provides the lower tester with a maximum of control over the upper tester which in turn is controlling and observing the upper interface of the IUT. The UT is built upon the "Glue Software" which aims to keep the UT independent from underlying service provider. 


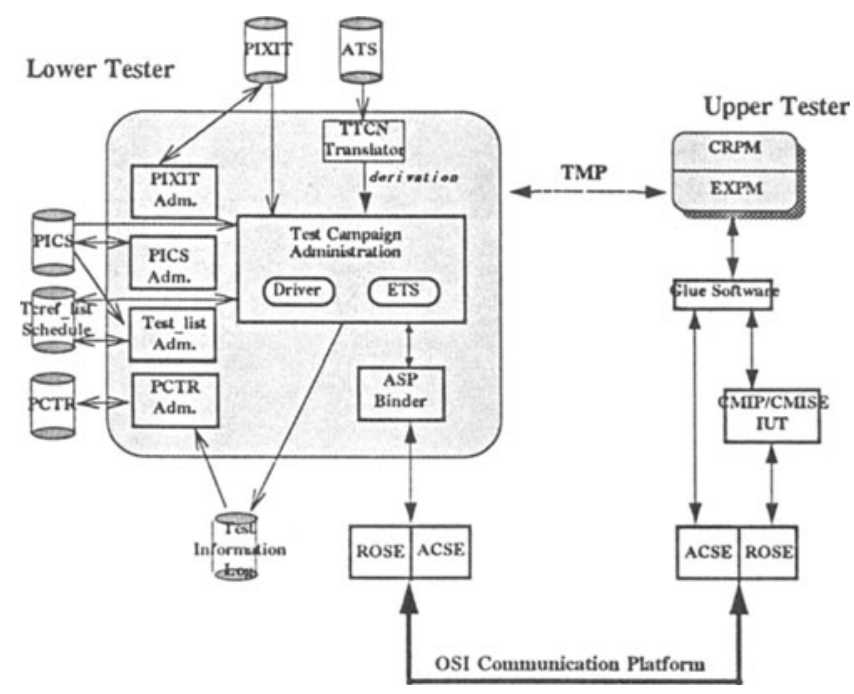

Figure 1. System Architecture of the CMIP CTS

\subsection{Architecture Description}

First, all the cylinders in Figure 1 represent the related information used during the conformance test process. For examples, ATS is the TTCN-based MP form for some protocol, and it will be used as the input for the TTCN translator. Clients who want to validate their products should supply the PICS (Protocol Implementation Conformance Statement) data. This information is used to specify which functionalities clients have implemented in their protocol products. The system will use the PICS data to automatically select test cases. Clients should also supply PIXIT (Protocol Implementation eXtra Information for Testing) data in order to parameterize the ETS. Tcref-list/Schedules is a data repository which contains test schedules and the list of references of selected test cases, where the test schedule is a group of test cases ready to be executed in a batch and the list of references of selected test cases is the list of test cases which are applicable to the client's implementation. PCTR (Protocol Conformance Test Report) is the official report about the test result for the client's product. Test Information Log is the data repository about the execution of the test cases with time stamp, abstract test events, and where necessary details about the PDU exchange for the protocol to be tested.

During the phase of the preparation for testing, test operator uses the TTCN translator to derive the ETS (Executable Test Suite) from the selected ATS. The PIXIT Adm. module and the PICS Adm. module are used to input and manage the client's information.

After the preparation phase, operator activates the Test Campaign Administration module which uses the PIXIT data to parameterize the ETS. He can select one individual test case or a test schedule generated by the Test_list Adm. module to execute. For every 
test case execution, the Test Campaign Administration module stores the execution information in the Test Information Log, and the ASP Binder realizes every send/receive behavior in the ETS by calling the corresponding service functions of those underlying protocol stacks. These send function calls will transmit the prepared PDUs to the IUT, and the IUT is supposed to respond appropriately. Finally, test operator activates the PCTR Adm. module to generate the final test report.

\section{Supporting Tools}

\subsection{TTCN Translator}

The TTCN translator plays a very important role in the test environment. It can be reused to derive the ETS for any TTCN-based ATS. There are two forms of TTCN, GR (GRaphical) form and MP (Machine Processable) form. GR form is easier for users to read, write and modify, while MP form is more suitable for a computer to process. These two forms are semantically equivalent. The TTCN translator is based on the IS (International Standard) version of TTCN. It reads the MP form of a TTCN-based ATS which may be produced by TIME, and then checks the syntax and semantics of the ATS. If its syntax and semantics are correct, the TTCN translator will generate the corresponding ETS which is written in $\mathrm{C}$ language.

The translator generates one $C$ routine for each test case or test step such that user can select individual test case to execute. About the ASN.1 definition parts of ATS, the TTCN translator passes them to pepsy, one tool of ISODE, to generate the corresponding $\mathrm{C}$ data structures file and the encoding/decoding routines which are used when LT sends/receives PDUs to/from the underlying protocol stacks.

In order to keep the generated ETS independent from the physical, underlying protocol stacks, we propose a programming interface, the send and receive function in the ETS. They are just function calls with necessary parameters, and the ASP binder shall realize their operations. So the underlying communication platform could be changed easily by replacing the ASP binder without regenerating the ETS.

The format of the send function is:

send( PCO name, ASP name, pointer to constraint).

The first parameter is the PCO (Point of Control and Observation) name. The ASP binder sends PDUs through this observation point. The second parameter is the ASP (Abstract Service Primitive) name. The final parameter is a pointer to a constraint which is a data structure containing values for the corresponding service primitive. Then the ASP binder has to extract values from those individual fields of the sending constraints, performs the necessary transformation, and calls the corresponding service functions of underlying protocol stacks, e.g., ACSE or ROSE in the CTS.

The receive function call needs no parameters. Its format is:

receive(). 
When the receive function is called, the ASP binder has to check if it has received any PDU (Protocol Data Unit) from the underlying stacks, and then transforms it into the corresponding data structure and inserts this structure into a queue for matching .

\subsection{TIME (TTCN-Based Integrated Management Environment)}

As the OSI protocols are becoming more and more complex, the sizes for the corresponding test suites are dramatically increased. So the development of test suites is a complex, error-prone and demanding process. This situation results in the requirement of such a software tool which can provides the significant improvement on productivity and reliability of ATS production. The TIME system is a tool to support the development of TTCN-written ATS.

TIME can be seen as a collection of some relevant tools. All these tools can be accessed through a friendly GUI (Graphic User Interface). We now introduce its functionalities by navigating through its important modules. The Importer and Exporter are used for interfacing with other vendor products. The Importer imports ATS in ISO standard TTCN.MP form, and translates the input into internal form for further manipulation. The Exporter performs the reverse operation, translating the ATS in internal representation into a TTCN.MP file with indentation. While conventional text editors see a TTCN ATS as a flat mass of text, TIME provides a syntax-directed table editor. The Table Editor displays the TTCN table proformas and facilitates the user to edit the contents of them without knowing the verbose and tedious keyword wrapping. The Table Printer is a very useful tool for printing TTCN.GR tables in a fashion style. Since most ATSs designed for OSI protocols contain hundreds of test cases, it is impractical to expect a single designer to write and verify the whole test suite. So, through the Concurrent Access Manager, the TIME system provides an environment for multiple users to concurrently access the same test suite from independent workstations. The main function of the Concurrent Access Manager is to assure users that data consistency is properly maintained.

\section{System Evaluation}

This section evaluates the system from different points of view.

\subsection{An Integrated Environment}

The system provides not only some useful tools but also a convenient and integrated environment, where users can easily edit their TTCN-based ATSs by benefiting from the TIME system. Users can use the TTCN translator to derive the ETS directly from the ATS, and develops the CTS by combining the ETS with its ASP binder. They can easily incorporate the new developed CTS in the system and easily operate the CTS because the system has a common flexible architecture, and provides a consistent user look-and-feel and operation convention. 


\subsection{High Productivity}

In the process of the development of ATS, the concurrent access functionality of TIME is highly appreciated. It facilitates a group of developers to simultaneously develop the ATS, and TIME provides a syntax-directed table editor. These features significantly improve productivity.

\subsection{Effectiveness and Robustness}

Before the completion of the development of the TTCN translator, the ATS is studied and the test case is manually coded one-by-one. It was very exhaustive and hard to debug and maintain these codes. With the aid of the TTCN translator, users can easily and quickly derive the whole ETS from the ATS. Furthermore, these codes are less errorprone and easier to maintain.

\section{Conclusion}

Up to now, there is no commercial CMIP CTS product which is based on the recommendation of the CPS Forum to provide users an open architecture and a consistent operating environment. The development of the CMIP CTS follows the recommendation of the CPS Forum. Besides, it can directly derive the ETS from the ATS which is written in IS version TTCN. Many test cases have been performed to test against the OSIMIS IUT. These test cases consists of basic connection testing, capacity testing and behavior testing. The OSIMIS IUT passes all these tests.

The test method used in the CMIP/CMISE ATS is the Coordinated Test Method. It requires the venders who provide their products for conformance testing to expose the application interfaces of their products in order to build the UT upon them. If the venders couldn't provide these application interfaces, a different test method must be used. Fortunately, the CTS3 NM project considers developing another ATS which uses the remote test method instead of the Coordinated Test Method.

\section{Terms and Abbreviations}

$\begin{array}{ll}\text { ASP } & \text { Abstract Service Primitive } \\ \text { ATS } & \text { Abstract Test Suite } \\ \text { BER } & \text { Basic Encoding Rule } \\ \text { CAP } & \text { Conformance Assessment Process } \\ \text { CMIP } & \text { Common Management Information Protocol } \\ \text { CMISE } & \text { Common Management Information Services } \\ \text { CTS } & \text { Conformance Test System } \\ \text { ETS } & \text { Executable Test Suites } \\ \text { LT } & \text { Lower Tester } \\ \text { PCO } & \text { Point of Control and Observation } \\ \text { PCTR } & \text { Protocol Conformance Test Report } \\ \text { PDU } & \text { Protocol Data Unit }\end{array}$




$\begin{array}{ll}\text { PICS } & \text { Protocol Implementation Conformance Statement } \\ \text { PIXIT } & \text { Protocol Implementation eXtra Information for Testing } \\ \text { SCTR } & \text { System Conformance Test Report } \\ \text { TCs } & \text { Test Centers } \\ \text { TIME } & \text { TTCN-based Integrated Management Environment } \\ \text { TMP } & \text { Test Management Protocol } \\ \text { TTCN } & \text { Tree and Tabular Combined Notation } \\ \text { TTCN.MP } & \text { TTCN Machine Processable } \\ \text { UT } & \text { Upper Tester }\end{array}$

\section{REFERENCES}

1. CPS Forum, The CPS Forum Technical Framework Specification, Volume I.

2. The second release of CMIP/CMISE Abstract Test Suite of CTS3 NM.

3. ISO/IEC 9595: 1991, Information Technology- Open Systems InterconnectionCommon Management Information Service Definition. CCITT Recommendation X.710(1991), Common Management Information Service Definition for CCITT applications- General concepts.

4. CCITT Recommendation X.711| ISO/IEC 9596-1: 1991( E), Information Technology- Open Systems Interconnection- Common Management Protocol Specification- Part 1: Specification, Edition 2.

5. ISO/IEC 9646-3: 1991, Information Technology- Open System InterconnectionConformance testing methodology and framework- Part 3: The Tree and Tabular Combined Notation.

6. CTS3 NM, the Establishment of a European Community Testing Service for Network Management, Annex F Test Management Protocol.

7. ISO/IEC 9646-1, Information Processing Systems - OSI Conformance Testing Methodology and Framework - Part 1: General Concepts.

8. ISO/IEC 9646-2, Information Processing Systems - OSI Conformance Testing Methodology and Framework - Part 2: Abstract Test Suite Specification.

9. ISO/IEC JTC1/SC21, OSI Conformance Testing Methodology and Framework Part 3: The Tree and Tabular Combined Notation( TTCN), ISO DIS 9646-3, Dec. 1989.

10. Ching-Sung LU, "TIME: A TTCN-Based Integrated Management Environment for Developing Standardized Conformance Test Suites." Proceedings of International Symposium on Communication, Vol.1 1991, pp. 376 380. 